\title{
Future Accelerator Magnet Needs
}

\author{
Arnaud Devred, Stephen A. Gourlay, and Akira Yamamoto
}

\begin{abstract}
Superconducting magnet technology is continually evolving in order to meet the demanding needs of new accelerators and to provide necessary upgrades for existing machines. A variety of designs are now under development, including high fields and gradients, rapid cycling and novel coil configurations. This paper presents a summary of $R \& D$ programs in the EU, Japan and the USA. A performance comparison between $\mathrm{NbTi}$ and $\mathrm{Nb}_{3} \mathrm{Sn}$ along with fabrication and cost issues are also discussed.
\end{abstract}

Index Terms-Accelerator, superconducting magnets.

\section{INTRODUCTION}

$\mathbf{F}$ OR over 50 years superconducting magnets have been a major enabling technology in the development of particle accelerators. The adventure started in the mid-1960's, thanks to the pioneering work of W.B. Sampson at BNL, who built a 76-mm-aperture, $85-\mathrm{T} / \mathrm{m}$ quadrupole magnet model wound from $\mathrm{Nb}_{3} \mathrm{Sn}$ ribbons and cold tested in January 1966 [1]. The feasibility and reliability of large superconducting magnet systems was demonstrated by the Tevatron at FNAL, which was commissioned in 1983 [2]. The Tevatron paved the way to commercial applications of applied superconductivity (such as Magnetic Resonance Imaging or MRI systems) and to a mini-series of ever more ambitious projects (HERA, SSC, UNK, RHIC and now LHC), which have continuously pushed the technology forward.

Since the time of the Tevatron, significant progress has been made in the design and production of superconducting accelerator magnets, enabling a gain of a factor $\sim 2$ in terms of dipole field. From an R\&D point of view, the largest ongoing accelerator magnet project, the LHC (that, among others, call for 1232 14.2-m-long, 56-mm-twin-aperture, 8.33 $\mathrm{T}$ arc dipole magnets), is complete [3]. So, it seems appropriate to pose the question: what else is there?

\section{CONDUCTOR OPTIONS}

Though there are a vast number of known superconducting materials, the number of viable options for magnet builders is limited to a select few. The choice of superconductor used in

Manuscript received October 5, 2004. This work was supported in part by the European Community-Research Infrastructure Activity under the FP6 "Structuring the European Research Area" program (CARE, Contract RII3-CT-2003506395) and by the Director, Office of Science, Office of High Energy Physics, Division of High Energy Physics, U.S. Department of Energy under Contract DE-AC03-76SF00098.

A. Devred is with CEA/Saclay, DSM/DAPNIA/SACM, 91191 Gif-surYvette, France and CERN, Accelerator Technology Department, CH-1211 Geneva 23, Switzerland (e-mail: arnaud.devred@cern.ch).

S. A. Gourlay is with the Lawrence Berkeley National Laboratory, Berkeley, CA 94720 USA (e-mail: sagourlay@lbl.gov).

A. Yamamoto is with the High Energy Accelerator Research Organization (KEK), Tsukuba 305-0801, Japan (e-mail: akira.yamamoto@kek.jp).

Digital Object Identifier 10.1109/TASC.2005.849530 magnets has significant impact on both performance and cost. There has been considerable progress within the past decade in the improvement of existing materials and the development of new candidates [4], [5].

\section{A. $\mathrm{NbTi}$}

$\mathrm{NbTi}$ has been, and still is the most predominant superconducting material in use. With critical parameters such as $B_{\mathrm{C} 2}$ $(0 \mathrm{~K}) \sim 14 \mathrm{~T}$ and $T_{\mathrm{C}}(0 \mathrm{~T}) \sim 9.5 \mathrm{~K}$ dipoles made with $\mathrm{NbTi}$ can reach operating fields of $\sim 7 \mathrm{~T}$ at $4.5 \mathrm{~K}$ and up to $9 \mathrm{~T}$ at $1.9 \mathrm{~K}$. NbTi has excellent mechanical properties and small filament diameters that are available in state-of-the art composites allowing operation over a large dynamic range. The cost per Tesla-meter of NbTi is also the lowest of the available superconductors due to large-scale industrial production. Over the past several decades NbTi accelerator magnets have been massproduced in laboratories and in industry. Progress in the development of NbTi peaked in the 1980's during the SSC. The most recent innovation is the method called Artificial Pinning Centers (APC) where normal metal is co-reduced with the NbTi to form an array of pinning centers. This method has resulted in enhanced performance relative to conventional $\mathrm{NbTi}$, especially at lower fields, as needed in MRI magnets [6]-[9].

Ternary NbTiTa alloys have the potential to provide a small gain in field (up to $1.25 \mathrm{~T}$ at $1.8 \mathrm{~K}$ ) while keeping similar wire and identical magnet manufacturing procedures. However, very little R\&D has been carried out on this material for the last 20 years and the potential for increased high-field performance was never fully translated in terms of critical current density, so that the investment needed to bring this technology to maturity may not seem worthwhile.

\section{B. $\mathrm{Nb}_{3} \mathrm{Sn}$}

$\mathrm{Nb}_{3} \mathrm{Sn}$ has a $B_{\mathrm{C} 2}$ and $T_{\mathrm{C}}$ that are about a factor of two greater than NbTi. These properties make it the current favorite for applications requiring high fields and/or high heat load operation. However, that advantage is countered by the brittle nature of the material and sensitivity to strain. $\mathrm{Nb}_{3} \mathrm{Sn}$ has a $B_{\mathrm{C} 2}(4.2 \mathrm{~K})$ $\sim 23-24$ Tesla and $T_{\mathrm{C}}(0 \mathrm{~T}) \sim 18 \mathrm{~K}$.

There are two fabrication techniques being explored: windand-react and react-and-wind. Wind-and-react is most widely used since it allows a variety of cross section geometries and reduces handling of the brittle material. Concern for the difficulty of producing long coils without damaging the coils has maintained interest in development of react-and-wind techniques, but coil geometries are limited to large bend radii. The use of $\mathrm{Nb}_{3} \mathrm{Sn}$ conductor typically results in significant coil magnetization effects in high field magnets due to large effective filament diameters [5]. A simple passive correction technique based on iron strips installed in the magnet bore or inside the magnet coil 


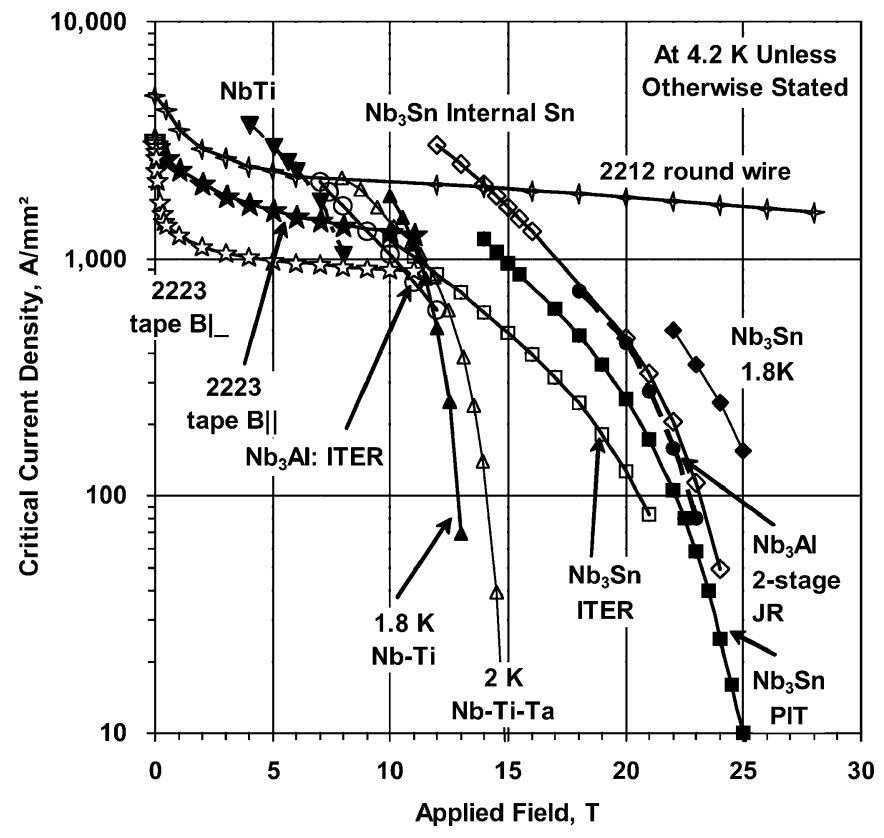

Fig. 1. Current density comparison (courtesy of P.J. Lee).

has been developed in order to reduce this effect [10]. This approach might lead to a significant increase in the dynamic range of $\mathrm{Nb}_{3} \mathrm{Sn}$ accelerator magnets and relax the requirements on the effective filament size in $\mathrm{Nb}_{3} \mathrm{Sn}$ strands.

\section{C. $\mathrm{Nb}_{3} \mathrm{Al}$}

$\mathrm{Nb}_{3} \mathrm{Al}$ has a potential for higher field operation and less strain sensitivity than $\mathrm{Nb}_{3} \mathrm{Sn}$. Programs in Japan are actively developing processing techniques for this material [11], [12]. Optimization of a process developed by the National Institute for Materials Science (NIMS), has resulted in a steady increase of $B_{\mathrm{C} 2}, T_{\mathrm{C}}$, and $J_{\mathrm{C}}$ [13]. It has been possible to increase the piece-length of the conductor by a factor of eight (to $2600 \mathrm{~m}$ ) with regard to enlarging the multifilament billet size up to $50 \mathrm{~kg}$. Stabilization techniques of ion-plating and/or electro-plating are pursued in order to apply a copper layer to the surface of the $\mathrm{Nb}_{3} \mathrm{Al}$ wire [14]. There are still significant manufacturing challenges to overcome in order to achieve properties competitive with $\mathrm{Nb}_{3} \mathrm{Sn}$.

Fig. 1 shows a comparison of current densities achieved for materials available in long lengths, including $\mathrm{Nb}_{3} \mathrm{Sn}$ and $\mathrm{Nb}_{3} \mathrm{Al}$ [15], [16].

\section{HTS}

Available HTS materials include BSCCO-2212, with a $T_{\mathrm{C}}(0 \mathrm{~T}) \sim 85 \mathrm{~K}$, and BSCCO-2223, with a $T_{\mathrm{C}}(0 \mathrm{~T}) \sim 110 \mathrm{~K}$. There has been steady progress in the development of these materials and kilometer-length quantities have been made in the form of multifilament tapes or round strands [4]. Rutherford cables have been successfully produced from $0.8 \mathrm{~mm}$ strands [17]. Due to the complex reaction process, react-and-wind is assumed to be the only coil winding alternative with the possible exception of small coils where wind-and-react might be used. Very high critical current densities have been achieved at high fields in YBCO short samples. However, commercial pro-
TABLE I

TARGET PARAMETERS FOR THE US AND THE EU CARE/NED $\mathrm{Nb}_{3} \mathrm{Sn}$ WIRE DEVELOPMENT PROGRAMS

\begin{tabular}{lll}
\hline \hline & US & CARE/NED \\
\hline$J_{\mathrm{C}}$ (non-copper) & $3,000 \mathrm{~A} / \mathrm{mm}^{2}$ & $1,500 \mathrm{~A} / \mathrm{mm}^{2}$ \\
& $@ 12 \mathrm{~T}, 4.2 \mathrm{~K}$ & $15 \mathrm{~T}, 4.2 \mathrm{~K}$ \\
$J_{\mathrm{e}}(12 \mathrm{~T}, 4.2 \mathrm{~K})$ & $1,000 \mathrm{~A} / \mathrm{mm}^{2}$ & $<50$ microns \\
Effective filament & $<40 \mathrm{microns}$ & \\
diameter & & $1.250 \mathrm{~mm}$ \\
Wire diameter & $0.3 \mathrm{~mm}-1.0 \mathrm{~mm}$ & $>200$ \\
RRR (after full reaction) & & $>50 \mathrm{~kg}$ \\
Process unit size & $100 \mathrm{~kg}$, \\
& $\begin{array}{l}\text { average piece length } \\
\end{array}$ & \\
Wire cost & $<\$ 1.50 / \mathrm{kA}-\mathrm{m}$ \\
& $(12 \mathrm{~T}, 4.2 \mathrm{~K})$ \\
Heat treatment time & $\begin{array}{l}\text { Max } 400 \mathrm{hrs} ; 50 \mathrm{hrs} \text { wind } \\
\text { and react }\end{array}$ \\
\hline \hline
\end{tabular}

duction of this material lags well behind BSCCO. The cost of all HTS material is very high and the production rate at present is too low. HTS materials exhibit tantalizing properties with the potential to produce much higher fields if the engineering drawbacks of low current density, strain sensitivity, reaction and industrialized cost can be overcome.

E. $\mathrm{MgB}_{2}$

The recent discovery of the superconducting properties of $\mathrm{MgB}_{2}[18]$ has led to a great deal of excitement. $\mathrm{MgB}_{2}$ is highly anisotropic but film studies have shown that, even in the "weak" direction, $B_{\mathrm{C} 2}(0)$ can reach $40 \mathrm{~T}$ [19] and current densities as high as $10^{5} \mathrm{~A} / \mathrm{cm}^{2}$ have been obtained at low temperatures and fields [20]. At this point, $\mathrm{MgB}_{2}$ is far from competing with $\mathrm{Nb}_{3} \mathrm{Sn}$, but for applications at temperatures below $25 \mathrm{~K}$ it has the potential to do so in the future. Since the raw material cost is low, the main challenge is developing the manufacturing process to yield a high performance conductor at low cost. A recent survey of these efforts can be found in [21].

\section{F. Conductor Development Programs}

There are several conductor development programs for HEP applications around the world. A program initiated by the U.S. Department of Energy in 1999 has resulted in a 50\% increase in noncopper critical current density of $\mathrm{Nb}_{3} \mathrm{Sn}$ wires to over $3,000 \mathrm{~A} / \mathrm{mm}^{2}$ at $12 \mathrm{~T}$ and $4.2 \mathrm{~K}$ [22]. A parallel program, recently initiated in the European Union, is part of the Next European Dipole (NED) activity of the Coordinated Accelerator Research in Europe (CARE) project [23], [24]. The aim of NED is to promote the development of large diameter wires (up to $1.25 \mathrm{~mm}$ ) with a noncopper critical current density of $1,500 \mathrm{~A} / \mathrm{mm}^{2}$ at $15 \mathrm{~T}$ and $4.2 \mathrm{~K}$, by at least two methods: Enhanced Internal Tin (EIT) and Powder in Tube (PIT). Goals for the two programs are compared in Table I.

In Japan, a method called Distributed Tin (DT) for $\mathrm{Nb}_{3} \mathrm{Sn}$ conductor has successfully produced wires with noncopper critical current density greater than $2,100 \mathrm{~A} / \mathrm{mm}^{2}$ at $12 \mathrm{~T}$ and $4.2 \mathrm{~K}$ 
and effective filament diameters of less than 60 microns [25]. The DT process may also more easily enable the achievement of a higher Residual Resistance Ratio (RRR). A research program on $\mathrm{Nb}_{3} \mathrm{Al}$ wires relying on the Rapid-Heating Quenching Transformation process (RHQT) is also being carried out for future accelerator magnet applications as a collaboration between KEK and NIMS [14], [16],

\section{High Field MAGNeTS}

\section{A. Quadrupoles for Interaction Regions}

The performance, or physics "reach" of a colliding beam accelerator is determined by the energy of the particles and the luminosity, where, for a given energy, the luminosity is a measure of the production rate for a particular process. The particles are brought to collision in the Interaction Regions (IR's) by sets of quadrupoles placed on either side of the collision point. As machines go to higher energies, there is a trend toward higher gradients. In many cases, it is aperture that dominates the requirements, leading to higher fields on the conductor. Also, the high radiation/high heat load environments in which these magnets are expected to operate, make them very challenging.

1) LHC Luminosity Upgrade: In a few years, the Large Hadron Collider (LHC) will become the world's highest energy accelerator. Under nominal running conditions, the experiments should reach their statistical limit in about 7 years. This time period also coincides with the expected lifetime of the IR magnets due to the high radiation environment. Plans are now being made to extend the physics reach of the LHC by first replacing the Interaction Region (IR) magnets to increase the luminosity, followed some years later by implementing a comprehensive plan to increase the energy [26], [27]. Assuming the performance of the machine follows the expected profile, new IR magnets would be needed sometime between 2015 and 2017. Replacement of the existing inner triplets is a milestone toward higher luminosity.

A first step on this road could be a luminosity recovery scheme based on NbTi technology [28]. However, to gain an order of magnitude on the present LHC nominal luminosity, it is necessary to rely on $\mathrm{Nb}_{3} \mathrm{Sn}$ technology. For these ultra-luminosity schemes, there are two fundamental inner triplet design options [29]: a large-aperture, single-bore inner triplet followed by beam separation dipoles or double-bore inner triplet with separation dipoles first. The R\&D programs target dipoles with a bore field of $\sim 15 \mathrm{~T}$, and an aperture of at least $88 \mathrm{~mm}$. The quadrupoles have a field gradient of $\sim 200 \mathrm{~T} / \mathrm{m}$, and an aperture $\sim 100 \mathrm{~mm}$. The magnets must operate in the extreme radiation environment generated by the interactions. These requirements emphasize the need for development of new technology.

a) LARP: In 2003, the US Department of Energy started the LHC Accelerator Research Program (LARP), a collaboration of BNL, FNAL, LBNL and SLAC directed toward the development of accelerator technology to fully utilize the physics potential of the LHC by maximizing the luminosity in the initial configuration and providing for a luminosity upgrade [30]. The program includes accelerator physics, commissioning, collimation, instrumentation and magnet R\&D. The principal goal of the magnet program is to produce designs for IR quadrupoles and dipoles relying on the highest performance superconducting materials. The aim is to extend and quantitate the limits on key performance parameters, providing accelerator physicists with IR options that most efficiently address the beam dynamics issues that limit machine operation. Some preliminary work on a variety of quadrupole and dipole designs has been done by LBNL, FNAL and BNL [31]-[33].

Recent progress in the development of $\mathrm{Nb}_{3} \mathrm{Sn}$ has encouraged the prospects for use in accelerator magnets but the application for the LHC upgrade adds additional issues to the already formidable list. The magnets will operate in a high radiation environment, subject to unprecedented beam induced heat loads, that will require development of radiation hard materials for coil construction and understanding the heat transfer characteristics of composite coils. The design process will necessitate working closely with accelerator physicists to understand trade-offs between magnet performance limits and IR upgrade options.

b) Next European Dipole (NED): In 2003, the European Union approved, after peer review and amendment, the Coordinated Accelerator Research in Europe (CARE) project, which, among others, includes a Joint Research Activity (JRA) nicknamed Next European Dipole (NED). The NED JRA is aimed at the development of a large-aperture $(88 \mathrm{~mm})$, high-field (15 T conductor peak field) $\mathrm{Nb}_{3} \mathrm{Sn}$ dipole magnet, that will serve as a technology test bed for LHC upgrades and that could be implemented in an existing superconducting cable test facility at CERN presently limited to $10 \mathrm{~T}$. The activity is divided up into two phases. The first phase, fully funded through CARE, encompasses the conductor development program described in Section II-F, and some limited studies on magnet design and conductor insulation. The second phase, for which funding is not yet secured, groups together all the tasks related to the detailed design, manufacturing and test of the magnet model.

The NED activity is supported by eight institutes throughout Europe: CCLRC/RAL, United Kingdom, CEA/DSM/DAPNIA, France, CERN, International, CIEMAT, Spain, INFN/Genova and INFN/Milano, Italy, Twente University, the Netherlands and Wroclaw University of Technology, Poland. It was launched on January 1st, 2004 and the first phase is expected to be completed by the end of 2006. Details on the program can be found in [24].

2) Linear Collider IR: A linear collider has been proposed as the logical choice to follow and complement the LHC. As for circular colliders, high gradient quadrupoles that operate in challenging environments are necessary.

c) TESLA: The Technical Design Report (TDR) of the Tera Electron volt Superconducting Linear Accelerator (TESLA) calls for a set of four final focusing quadrupole magnets with a $250 \mathrm{~T} / \mathrm{m}$ gradient and a $56 \mathrm{~mm}$ aperture. An additional requirement is that some of these magnets are localized inside the detector magnet and must operate in a $4 \mathrm{~T}$ solenoidal background field.

CEA/Saclay has produced a quadrupole magnet design with the required gradient and aperture leading to a peak field on the conductor of 8.6 T. In order to have sufficient operating margin, $\mathrm{Nb}_{3} \mathrm{Sn}$ must be used. Another issue is the large and complex force distribution due to the presence of a background field. CEA/Saclay is presently manufacturing a model of such a magnet, relying on a cable made up of ITER-HPI-like, $\mathrm{Nb}_{3} \mathrm{Sn}$ 
strands, that is scheduled to be tested in late 2005 in the aperture of a 2-T MRI magnet [34].

d) Compact quadrupole magnets: Other designs of linear collider IR's call for large beam crossing angles. In such configurations, the final focusing quadrupole magnets must be very compact so as to clear the way radially for the crossing beam. Preliminary design studies, carried out mostly at BNL, have shown that there might be some interest in relying on superconducting magnet technology [35].

\section{B. Collider Dipoles}

Magnet applications for future colliding beam machines can be separated into two categories; upgrades of existing facilities such as the LHC, described below, and new facilities. High fields are the obvious choice for an upgrade scenario, while other factors such as tunnel cost, cryogenics, logistics and location are factors that have to be considered along with magnet performance and cost at a green-field site.

Cost is more of an issue for colliders than other applications given the large number of magnets required. Many programs around the world are engaged in activities that directly contribute to the development of technology for collider magnets. In particular, the addition of $\mathrm{Nb}_{3} \mathrm{Sn}$ to the list of viable superconductors has lead to an unprecedented number of options. A summary of the most recent dipole work and related issues can be found in [36].

The second phase of the LHC upgrade proposal is to increase the energy in the existing LHC tunnel [26]. This will require major changes to the LHC arcs (new dipoles and quadrupoles) as well as the injector chain. A factor of two increase in energy implies an operating dipole field of at least $16 \mathrm{~T}$ and quadrupoles with a gradient of $450 \mathrm{~T} / \mathrm{m}$ and aperture of $\sim 50 \mathrm{~mm}$. A program to develop these magnets may take 15-20 years and will necessarily focus on cost as well as technology.

\section{FAST-CyCLING MAGNETS}

Until recently, there have been few applications of fast-pulsed superconducting accelerator magnets. One example is the dipole design used for the Nuclotron at JINR in Dubna [37]. Despite the complications of field quality degradation and cryogenic losses caused by persistent currents in the superconductor and eddy currents in the cable, iron and surrounding structures, the increasing demand for high intensity beams requires superconducting magnets with high-ramp-rate-capability. Note that all the designs considered for this purpose rely on NbTi technology.

\section{A. GSI}

The proposed international Facility for Antiproton and Ion Research (FAIR) at GSI, consists of two synchrotrons in one tunnel, the SIS 100 (100 T-m rigidity) and SIS 300 (300 T-m rigidity) [38]. The SIS 100 is the heart of the machine, accelerating protons and ions at a high repetition rate and distributing them to other parts of the complex. The required dipole ramp rate is $4 \mathrm{~T} / \mathrm{s}$ up to a field of about $2 \mathrm{~T}$ for SIS 100 with a $1-\mathrm{Hz}$ duty cycle and $1 \mathrm{~T} / \mathrm{s}$ up to $6 \mathrm{~T}$ for SIS 300, with a duty cycle of $50 \%$. Each magnet has particular technical challenges.

R\&D on the SIS 100 magnet started with a study of possible improvements of the Nuclotron dipole. Designs based on both $4 \mathrm{~K}$ and $80 \mathrm{~K}$ iron yokes are being pursued. Significant progress has been made in the reduction of $\mathrm{AC}$ losses in the yoke and coil structures [39], [40]. A low-loss, efficiently cooled and stable conductor is necessary for reliable operation over a projected 200-million-cycle lifetime. A Cable-in-Conduit Conductor (CICC) has been proposed as an alternative to the $\mathrm{Nu}-$ clotron cable [41].

The original proposal of a SIS 200 using 4-T magnets has been replaced with the more challenging SIS 300, using 6-T magnets. Prior to the increase, a SIS-200 prototype based on the RHIC design was built with modifications to enable high ramp rates. Modifications included inserting stainless-steel cores into the cable to reduce inter-strand resistance, laser-cutting cooling holes in the polyimide insulation on the cable thin edge, and where possible, using components made from materials to reduce AC losses [42], [43]. The magnet has been operated successfully at $2 \mathrm{~T} / \mathrm{s}$. The higher field design required for the SIS 300 is based on the UNK 5 T [44] dipole combined with the successful elements of the RHIC-based SIS 200 prototype [45], [46]. The GSI R\&D program is a collaboration of several institutions. A review of the most recent work can be found in [47].

\section{B. SPS Upgrade}

A necessary consequence of an LHC energy upgrade is to increase the injection energy in order to limit the dynamic range (between injection and colliding energy) to less than 10 [48]. This would also benefit the current LHC and the various LHC IR upgrade schemes under consideration by reducing the effects of time-decay and snap-back of the low order multipole fields at injection, which requires sophisticated correction schemes. Two options are being considered. The first is the addition of a booster ring that would accelerate beam at $1 \mathrm{TeV}$ or higher. The magnets for this ring would have a bore field of 4 to $6 \mathrm{~T}$, an aperture between 80 and $100 \mathrm{~mm}$ and a ramp-rate of $1 \mathrm{~T} / \mathrm{s}$. This option also requires an upgrade of the beam transport system. The second option is to add a booster ring in the LHC tunnel that would accelerate beam from the existing SPS for injection into the LHC. The magnet requirements are more modest; field up to $2 \mathrm{~T}, 50 \mathrm{~mm}$ aperture and a ramp-rate of $0.1 \mathrm{~T} / \mathrm{s}$. A reasonable plan would be to pursue both options, benefiting from the $R \& D$ program to develop high ramp-rate magnets for GSI.

\section{Cost-Optimized Magnets}

\section{A. Combined Function}

A neutrino-oscillation experiment, nicknamed T2K (from Tokay to Kamioka), is being prepared at the Japan Proton Accelerator Research Complex (J-PARC) as a joint project between JAERI and KEK [49], [50]. A series of superconducting magnets are required for the arc section of the primary proton beam extracted from the $50 \mathrm{GeV}$ synchrotron. The proton beam is to be bent by 87 degrees and transported to the target to generate an intense neutrino beam aiming at the Super-Kamiokande detector located $295 \mathrm{~km}$ west of J-PARC.

A superconducting combined function magnet has been proposed to optimize the magnet performance with a strong emphasis on cost effectiveness [51], [52]. This magnet, which features a coil radius of $170 \mathrm{~mm}$, provides dipole $(2.6 \mathrm{~T})$ and 


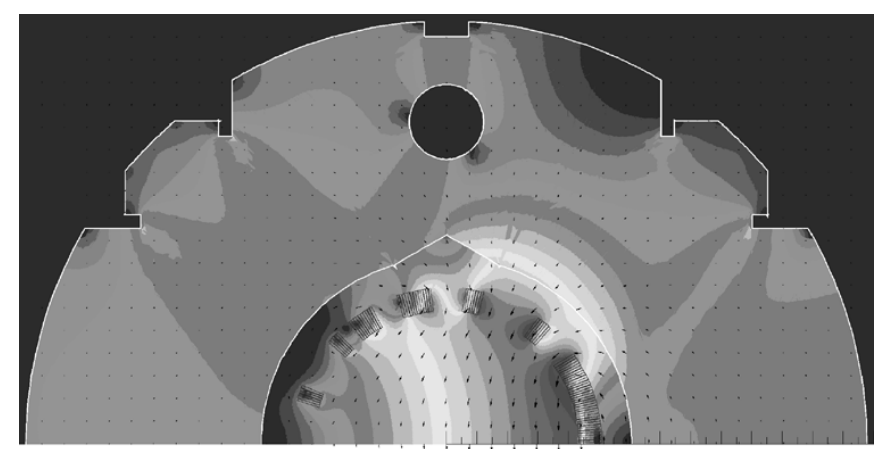

Fig. 2. Field map of combined-function magnet for J-PARC.

quadrupole $(19 \mathrm{~T} / \mathrm{m})$ fields superimposed in the same bore. The optics in the bending section of the beam line is simplified by using 14 pairs of these combined-function magnets. Also, it offers the advantage of only requiring a single component to design instead of separate dipoles and quadrupoles. The magnet design has been much simplified by the choice of a single layer coil surrounded by plastic collars and an iron, flux-return yoke. A prototype of a combined-function magnet is being developed at KEK [53]. Fig. 2 shows a cross section of this magnet.

\section{B. RIKEN Magnets}

Sector magnets for the Superconducting Ring Cyclotron (SRC) are under construction at RIKEN [54]. The SRC magnet system is made up of six sector magnets delivering a 3.8-T field. Each sector magnet is $7.2 \mathrm{~m}$ in length, $6 \mathrm{~m}$ in height and 800 tons in weight, with a sector angle of 25 degrees. For the first time in accelerator magnets, it was decided to rely on an aluminum-stabilized conductor on the grounds of cost optimization.

The Rutherford-type, $\mathrm{Cu} / \mathrm{NbTi}$ cable was co-extruded with high strength aluminum-alloy stabilizer. The components of the sector magnets as well as the cryogenics cooling system have been manufactured and the sector system assembly is in progress.

\section{Special Magnets}

\section{A. Wigglers and Undulators}

The demand for new sources of Synchrotron Radiation is growing, leading to the construction of new facilities and upgrades of existing facilities in order to provide higher performance beams. Light sources depend on undulators to provide high brilliance beams of tunable radiation. Short period undulators based on Permanent Magnet (PM) technology represent the state-of-the art. Performance beyond the limits imposed by permanent magnets requires superconducting technology. The superior magnetic properties of superconductors generate the potential for larger gaps, shorter periods and higher fields, substantially outperforming devices based on PM technology. Aside from the obvious issues associated with field quality, a major challenge is developing a cryogenic system that supports small gap operation in the presence of beam heating. In addition, there is a premium on cryogen-free operation using cryocoolers since most existing light sources do not have cryogenic infrastructure. An overview of current activity in this rapidly growing field can be found in the presentations at a recent workshop, hosted by the European Synchrotron Radiation Facility (ESRF) in June 2003 [55]. Work that pushes the state-of-the-art can be found in $[56]-[59]$.

\section{B. Helical Magnets}

Helical magnets are used to control particle spin. Several magnets of this type, referred to either as "Snakes" or "Rotators", depending on their helicity, angular orientation and particular field, have been built for RHIC and are being designed for the AGS [60], [61]. Aside from the unique coil geometry, the magnet construction also employs a novel winding process. The magnets are built by placing small diameter superconducting cables into helical slots milled into thick-walled aluminum cylinders. An iron yoke is placed around two nested coils and combined with 3 additional assemblies to form a Snake or a Rotator. For RHIC, the basic unit is a superconducting dipole producing a $4 \mathrm{~T}$ dipole field that rotates through 360 degrees in a length of 2.4-m. A Snake with a 3 T field rotating through 520 degrees and an aperture of $15 \mathrm{~cm}$ will be installed in the AGS next year.

\section{FFAG Magnets}

The Fixed Field Alternating Gradient (FFAG) accelerator concept is receiving renewed attention for many applications such as High Energy Physics experiments, electric power generation and cancer therapy. However, downsizing the accelerator is essential if it is to become widely used, especially for cancer therapy. The adoption of a superconducting magnet system has been proposed in order to miniaturize the accelerator [62]. The static magnetic field required for the FFAG accelerator is ideal for the application of superconducting magnet technology, and the high magnetic field makes the accelerator more compact for a given beam energy. The FFAG accelerator magnet requires a nonlinear magnetic field that increases with the $k$-th power of the orbit radius, where $k$ is the geometrical field index in the accelerator. A left/right asymmetric coil structure is being considered for an effective magnetic design to generate the FFAG field.

\section{RIA Fragment Separator Magnets}

The Rare Isotope Accelerator (RIA) is a proposed major facility in the United States for Nuclear Science research. One of the most challenging components of the facility is the fragment separator, used to select and transport isotopes to the experimental areas. Efficiency requirements dictate the use of superconducting magnets for at least the first focusing quadrupole triplet of the separator. The magnet requirements are modest in comparison with most superconducting magnet designs. There are several varieties but an $8 \mathrm{~T} / \mathrm{m}$ gradient, warm-bore aperture of $50 \mathrm{~cm}$ and $1 \mathrm{~m}$ in length is the most difficult. However, the magnets are exposed to several orders of magnitude more radiation and energy deposition than ordinary magnets receive during their entire lifetime. An approach using HTS coils in a superferric yoke is being developed by BNL [63]. The higher operating temperature $(20-30 \mathrm{~K})$ is an advantage but several technological issues including radiation tolerance of HTS conductors need to be investigated. 


\section{MAGNET PROGRAMS}

\section{A. Europe}

In addition to the NED program, which relies on a synergy of efforts among European laboratories [24], and to the GSI magnet R\&D program, which also calls upon an international collaboration [47], CEA/Saclay, in France, and Twente University, in The Netherlands, are also working on $\mathrm{Nb}_{3} \mathrm{Sn}$ magnets for future accelerator applications.

1) CEA/Saclay: CEA/Saclay has launched in the Fall of $1995 \mathrm{a} \mathrm{Nb}_{3} \mathrm{Sn} \mathrm{R} \& \mathrm{D}$ program that presently includes three main tasks: 1) conductor development, in collaboration with Alstom/MSA, 2) insulation development, in collaboration with the Laboratory of Ceramics and Advanced Materials of CEA/DAM and the European Institute for Membranes of Montpellier, France and 3) magnet development. Each task is articulated into two phases; Phase I is aimed at bridging the gap by relying on existing technological solutions already more or less known and proven in the rest of the world, while Phase II is aimed at developing innovative technological solutions meant to improve performance, facilitate industrialization, and lower costs and failure risks.

Regarding the conductor task, the goal of Phase I was to produce a $\mathrm{Nb}_{3} \mathrm{Sn}$ wire responding to specifications similar to the ITER-HP-I specifications (with a non-Cu $J_{\mathrm{C}}$ of $\sim 750 \mathrm{~A} / \mathrm{mm}^{2}$ at $4.2 \mathrm{~K}$ and $12 \mathrm{~T}$ ). It was achieved by Alstom/MSA in 1998 [64]. The goal of Phase II, which is still ongoing, is to achieve a non- $\mathrm{Cu} J_{\mathrm{C}}$ of $2000 \mathrm{~A} / \mathrm{mm}^{2}$ at $4.2 \mathrm{~K}$ and $12 \mathrm{~T}$. It has now become an intermediate step in the perspective of the NED conductor development program (see Section II-F). The Phase I of the insulation task was devoted to the development of a thin $(60 \mu \mathrm{m})$ quartz fiber tape, while Phase II is concerned with a ceramic-based, innovative insulation system [65], [66], Finally, Phase I of the magnet task includes the $\mathrm{Nb}_{3} \mathrm{Sn}$ quadrupole magnet model presently under construction that aims at the TESLA IR (see Section III-A-2-a) [34], while Phase II could be a significant contribution to the manufacturing of the NED magnet model.

2) Twente University: In 1998, Twente University signed a collaboration agreement with CERN to develop a 1-m-long, 88-mm-aperture $\mathrm{Nb}_{3} \mathrm{Sn}$ dipole magnet model with a nominal field of $10 \mathrm{~T}$ [67]. Such a magnet could be used to replace advantageously the low-field (4.5-T) D1 dipole magnets used to separate the colliding beams in the crowded LHC IR's. (Note that the parameters of this magnet were chosen so as to replace the existing D1 magnet while keeping the present IR optics and without referring to any specific IR or luminosity upgrade scenario.) The program was delayed due to problems in the cabling of the high-performance $\mathrm{Nb}_{3} \mathrm{Sn}$ PIT wires especially developed for this purpose [68]. The problems are now resolved, and plans are being discussed to complete the program.

\section{B. Japan}

The magnet program at KEK is focusing in two directions. The first is concerned with the development of advanced highfield conductors, such as $\mathrm{Nb}_{3} \mathrm{Sn}$ (Distributed Tin process), and $\mathrm{Nb}_{3} \mathrm{Al}$ (Rapid Quenching process), especially focusing on the 12-to-15-T field range.
The other stream is to develop cost-effective magnets for particle accelerators and beam transfer lines. The dipole-andquadrupole combined-function magnets enable the construction of beam lines that rely on a single-component design. The primary proton beam line of the J-PARC project is under construction. The combined-function magnet may also be a smart choice for the FFAG superconducting magnet system as well.

As a unique and innovative approach, high-strength, aluminum-stabilized superconducting cables are being used in the development of sector-type magnet components for the RIKEN SRC cyclotron.

\section{US}

The US magnet program represents a diverse and complementary approach to developing technology for high field magnets [69]. It is primarily focused on $\mathrm{Nb}_{3} \mathrm{Sn}$, with some attention to developing the potential of HTS.

1) Brookhaven National Laboratory: The BNL program has been involved with $\mathrm{Nb}_{3} \mathrm{Sn}$ for many years. Their current high field magnet program is centered on the react-and-wind approach for both $\mathrm{Nb}_{3} \mathrm{Sn}$ and HTS. Techniques for winding the brittle cable are studied using "10-turn coils". For the near future they are planning the fabrication of 12-T background coils for high field insert tests using react-and-wind $\mathrm{Nb}_{3} \mathrm{Sn}$ [70], [71].

2) Fermi National Accelerator Laboratory: The magnet program at FNAL is directed toward development of 10-12 T $\mathrm{Nb}_{3} \mathrm{Sn}$ accelerator dipoles for future hadron colliders. The program utilizes several approaches: Cosine-theta and block coils in the common coil configuration and both wind-and-react and react-and-wind fabrication techniques [72]. Several $1 \mathrm{~m}$ models have been built and tested. A recent test of a cosine-theta model using Powder-In-Tube (PIT) conductor reached a field of 10 Tesla at $2.2 \mathrm{~K}$ [73].

3) Lawrence Berkeley National Laboratory: The LBNL superconducting magnet program is directed toward advancing all aspects of the technological infrastructure for high field magnet development relevant to possible future accelerators. The program has produced dipole field records in threes geometries: D20, a 4-layer cosine-theta, reached a field of $13.5 \mathrm{~T}$ at $1.9 \mathrm{~K}$ [74], RD-3, a common coil, achieved $14.5 \mathrm{~T}$ at $4.5 \mathrm{~K}$ [75] and most recently, HD-1, a simple block magnet, reached $16.1 \mathrm{~T}$ at $3.3 \mathrm{~K}[76]$.

4) Texas A\&M University: The Texas A\&M program is based on high field, wind-and-react coils using internal structures to limit coil stress [77]. The current project is the construction of TAMU2 in which one of the three coil modules of a $12 \mathrm{~T}$ design is housed in the yoke structure designed for the complete dipole.

\section{CONCLUSIONS}

The LHC, which has dominated the magnet R\&D scene for almost two decades, is near completion. The international activities that have emerged from the shadow of this demanding project, while consisting of much smaller efforts, combines to form a diverse and innovative program of magnet development with exciting possibilities for future applications to accelerator technology. 


\section{ACKNOWLEDGMENT}

The authors would like to thank L. Bottura, D. Dietderich, E. Fischer, R. Gupta, M. Harrison, J. Kaugerts, P. Lee, A. McInturff, G. Moritz, T. Ogitsu, A. den Ouden, B. Parker, S. Prestemon, R. Scanlan, W. Scandale, P. Wanderer, E. Willen, A. Zlobin, and A. Zeller.

\section{REFERENCES}

[1] W. B. Sampson, "Superconducting magnets for beam handling and accelerators," in Proc. 2nd Int. Conf. Magn. Techn., H. Hadley, Ed., 1967, pp. 574-578.

[2] "A report on the design of the Fermi National Accelerator Laboratory superconducting accelerator," May, 1979.

[3] G. de Rijk, "Status report on the LHC main magnet production," to appear in these proceedings.

[4] R. M. Scanlan et al., "Superconducting materials for large scale applications," Proc. IEEE, vol. 92, no. 10, pp. 1639-1654, Oct. 2004.

[5] A. Devred, "Practical low temperature superconductors for electromagnets," CERN, Geneva, Switzerland, CERN Yellow Report 2004-006, July 12, 2004.

[6] G. L. Dorofejev et al., "Artificial pinning centers," in Proc. 9th Int. Conf. Magn. Techn., C. Marinucci and P. Weymuth, Eds., ETH, Zurich, 1985, pp. 564-566.

[7] L. D. Cooley and L. R. Motowidlo, "Advances in high field superconducting composites by addition of artificial pinning centers to niobiumtitanium," Supercond. Sci. Technol., vol. 12, pp. R135-R151, 1999.

[8] L. R. Motowidlo et al., "The pinning strength and upper critical fields of magnetic and nonmagnetic artificial pinning centers in Nb47wt\% Ti wires," IEEE Trans. Appl. Supercond., vol. 13, pp. 3351-3354, 2003.

[9] O. V. Chernyi et al., "The microstructure and critical current density of Nb-48 Wt.\%Ti superconductor with very high alpha-Ti precipitate volume and very high critical current," Adv. Cryo. Eng., vol. 48(B), pp. 883-890, 2002.

[10] V. V. Kashikhin and A. V. Zlobin, "Correction of the persistent current effect in Nb3Sn dipole magnets," IEEE Trans. Appl. Supercond., vol. 11, no. 1, pp. 2058-2061, 2001.

[11] K. Okuno et al., "Test of the NbAl insert and ITER central solenoid model coil," IEEE Trans. Appl. Supercond., vol. 13, pp. 1437-1440, 2003.

[12] T. Takeuchi, "Nb3Al conductors for high-field applications," Supercond. Sci. Technol., vol. 13, pp. R101-R119, 2000.

[13] N. Banno et al., "Multifilamentary Nb/Al-Ge and Nb/Al-Si precursor fabrication using the intermediately rapid heating and quenching technique," Supercond. Sci. Technol., vol. 17, pp. 320-326, 2004.

[14] T. Takeuchi and K. Tsuchiya, "Status and perspective of the $\mathrm{Nb}_{3} \mathrm{Al}$ development in Japan," in Proc. Workshop on Accelerator Magnet Superconductor (WAMS), Archamps, France, Mar. 22-24, 2004.

[15] K. Tsuchiya et al., "Development of $\mathrm{Nb}_{3} \mathrm{~A} 1$ superconducting wire for accelerator magnets," IEEE Trans. Appl. Supercond., vol. 14, no. 2, pp. 1016-1019, 2004.

[16] A. Yamamoto, "Advances in superconducting magnets for particle physics," IEEE Trans. Appl. Supercond., vol. 14, no. 2, pp. 477-484, 2004.

[17] T. Hasegawa et al., "HTS conductors for magnets," IEEE Trans. Appl. Supercond., vol. 12, no. 1, pp. 1136-1140, 2002.

[18] J. Nagamatsu et al., "Superconductivity at $39 \mathrm{~K}$ in magnesium diboride," Nature, vol. 410, pp. 63-64, 2001.

[19] A. Gurevich et al., "Very high upper critical fields in $\mathrm{MgB}_{2}$ produced by selective tuning of impurity scattering," Supercond. Sci. Technol., vol. 17, no. 2, pp. 278-286, Suppl. S 2004.

[20] S. Y. Xu et al., "High critical current density and vortex pinning of epitaxial $\mathrm{MgB}_{2}$ thin films," Phys Rev. B, vol. 68, pp. 224 501-224 508, 2003.

[21] R. Flukiger et al., "Superconducting properties of $\mathrm{MgB}_{2}$ tapes and wires," Physica C, vol. 385, pp. 286-305, 2003.

[22] R. M. Scanlan et al., "Development of cost-effective $\mathrm{Nb}_{3}$ Sn conductors for the next generation hadron colliders," Adv. Cryo. Eng., vol. 48B, pp. 933-940, 2002.

[23] A. Devred et al., "High field accelerator magnet R\&D in Europe," IEEE Trans. Appl. Supercond., vol. 14, no. 2, pp. 339-344, 2004.

[24] _ - "Status of the Next European Dipole (NED) activity of the Collaborated Accelerator Research in Europe (CARE) project," to appear in these proceedings.
[25] M. Wake et al., "Development of high current density $\mathrm{Nb}_{3} \mathrm{Sn}$ conductor with distributed tin configuration," Adv. Cryo. Eng., vol. 50B, pp. 410-416, 2004.

[26] O. Bruning et al., "LHC luminosity and energy upgrade: A feasibility study," CERN, Geneva, Switzerland, CERN LHC Project Report 626, Dec. 2002.

[27] F. Ruggiero et al., "LHC accelerator R\&D and upgrade scenarios," CERN, Geneva, Switzerland, LHC Project Report 666, 2003.

[28] — - "Performance limits and IR design of a possible LHC luminosity upgrade based on NbTi SC magnet technology," in Proc. 2004 Eur. Part. Accel. Conf., 2004, pp. 608-610.

[29] J. Strait et al., "Toward a new LHC interaction design for a luminosity upgrade," in Proc. 2003 Part. Accel. Conf., 2003, pp. 146-150.

[30] R. Kephart et al.. The U.S. LHC Accelerator Research Program: A Proposal. [Online] http://www-td.fnal.gov/LHC/USLARP.html

[31] G. Sabbi et al., " $\mathrm{Nb}_{3} \mathrm{Sn}$ quadrupole magnets for the LHC IR," IEEE Trans. Appl. Supercond., vol. 13, no. 2, pp. 1262-1265, 2003.

[32] A. V. Zlobin et al., "Conceptual design study of Nb3Sn low-beta quadrupoles for 2 nd generation LHC IRs," IEEE Trans. Appl. Supercond., vol. 13, no. 2, pp. 1266-1269, 2003.

[33] R. Gupta et al., "Open mid-plane dipole design for LHC IR upgrade," IEEE Trans. Appl. Supercond., vol. 14, no. 2, pp. 259-262, 2004.

[34] M. Durante et al., "Development and manufacturing of a Nb3Sn quadrupole magnet model at CEA/Saclay for the Tesla interaction region," IEEE Trans. Appl. Supercond., vol. 14, no. 2, pp. 357-360, 2004.

[35] B. Parker, private communication, 2002.

[36] A. den Ouden, "The challenges of $\mathrm{Nb}_{3} \mathrm{Sn}$ technology for accelerator magnets," to appear in these proceedings.

[37] A. D. Kovalenko, "Status of the nuclotron," in Proc. 1994 European Particle Accelerator Conf., 1994, pp. 161-164.

[38] W. Henning, "FAIR - an international accelerator facility for research with ions and antiprotons," in Proc. 2004 European Particle Accelerator Conf., 2004, pp. 50-53.

[39] E. Fischer et al., "Minimization of AC power losses in fast-cycling window frame $2 \mathrm{~T}$ superferric magnets with the yoke at $4.5 \mathrm{~K}$," to appear in these proceedings.

[40] A. Kovalenko et al., "Design and study of a superferric model dipole and quadrupole magnets for the GSI fast-pulsed synchrotron SIS 100," in Proc. 2004 Eur. Part. Accel. Conf., 2004, pp. 1735-1737.

[41] L. Bottura et al., "A cable-in-conduit superconductor for pulsed accelerator magnets," to appear in these proceedings.

[42] P. Wanderer et al., "Initial test of a fast-ramped superconducting model dipole for GSI's proposed SIS 200 accelerator," in Proc. 2003 Particle Accelerator Conf., 2003, pp. 2162-2164.

[43] M. N. Wilson et al., "Measured and calculated losses in model dipole for GSI's heavy ion synchrotron," IEEE Trans. Appl. Supercond., vol. 14, no. 2, pp. 306-309, 2004

[44] A. I. Ageev et al., "The development and study of superconducting magnets for the UNK," IEEE Trans. Magn., vol. 28, no. 1, pp. 682-685, 1992.

[45] J. Kaugerts et al., "Design of a $6 \mathrm{~T}, 1 \mathrm{~T} / \mathrm{s}$ fast-ramping synchrotron magnet for GSI's planned SIS 300 accelerator," to appear in these proceedings.

[46] A. Ageev et al., "Development of superconducting dipole design for creation of fast-cycling magnetic fields," IEEE Trans. Appl. Supercond., vol. 14, no. 2, pp. 295-299, 2004.

[47] G. Moritz, "Fast-pulsed SC magnets," in Proc. 2004 European Particle Accelerator Conf., 2004, pp. 132-136.

[48] W. Scandale, private communication, 2004.

[49] http://neutrino.kek.jp/jhfnu/ [Online]

[50] M. Furusaka et al., "The joint project for high-intensity proton accelerators," KEK Report 99-1; JAERI-Tech 99-056; JHF-99-3, 1999.

[51] T. Ogitsu et al., "Superconducting magnet system at the $50 \mathrm{GeV}$ proton beam line for the J-PARC neutrino experiment," IEEE Trans. Appl. Supercond., vol. 14, no. 2, pp. 604-607, 2004.

[52] T. Nakamoto et al., "Design of superconducting combined function magnets at the $50 \mathrm{GeV}$ proton beam line for the J-PARC neutrino experiment," IEEE Trans. Appl. Supercond., vol. 14, no. 2, pp. 616-619, 2004.

[53] - "Development of a prototype of superconducting combined function magnet for the $50 \mathrm{GeV}$ proton beam line for the J-PARC neutrino experiment," to appear in these proceedings.

[54] A. Goto et al., "Sector magnets for the RIKEN superconducting ring cyclotron," IEEE Trans. Appl. Supercond., vol. 14, no. 2, pp. 300-303, 2004.

[55] http://www.esrf.fr/Accelerators/Conferences/ID_Workshop [Online]

[56] S. H. Kim et al., "Design and development of a short-period superconducting undulator at the APS," to appear in these proceedings.

[57] D. R. Dietderich et al., "Design, fabrication and test results of undulators made with $\mathrm{Nb}_{3} \mathrm{Sn}$ cable," to appear in these proceedings. 
[58] S. Kubsky et al., "New cryogen free design for superconductive mini-gap undulators," to appear in these proceedings.

[59] B. Kostka et al., "Superconductive undulators with variable polarization direction," to appear in these proceedings.

[60] E. Willen et al., "Performance summary of the helical magnets for RHIC," in Proc. 2003 Particle Accelerator Conf., 2003, pp. 164-166.

[61] T. Roser et al., "Acceleration of polarized beams using multiple strong partial Siberian snakes," in Proc. 2004 European Particle Accelerator Conf., 2004, pp. 1577-1579.

[62] T. Obana et al., "Magnetic field design of a superconducting magnet for the FFAG accelerator," to appear in these proceedings.

[63] R. Gupta et al., "Radiation resistant HTS quadrupoles for RIA," to appear in these proceedings.

[64] M. Durante et al., "Development of a $\mathrm{Nb}_{3} \mathrm{Sn}$ multifilamentary wire for accelerator magnet applications," Physica C, vol. 354, pp. 449-453, 2001.

[65] A. Devred, "Insulations systems for $\mathrm{Nb}_{3} \mathrm{Sn}$ accelerator magnet coils manufactured by the wind \& react technique," IEEE Trans. Appl. Supercond., vol. 12, no. 1, pp. 1232-1237, 2002.

[66] A. Puigségur et al., "Development of an innovative insulation for $\mathrm{Nb}_{3} \mathrm{Sn}$ wind \& react coils," Adv. Cryo. Eng. (Materials), vol. 50, pp. 266-272, 2003.

[67] A. den Ouden et al., "A $10 \mathrm{~T} \mathrm{Nb}_{3} \mathrm{Sn}$ model separator dipole magnet for the CERN LHC," in Proc. 15th Int. Conf. Mag. Technol.. Beijing, China: Science Press, 1998, pp. 137-140.
[68] — - "Progress in the development of an 88-mm bore $10 \mathrm{~T} \mathrm{Nb}_{3} \mathrm{Sn}$ dipole magnet," IEEE Trans. Appl. Supercond., vol. 11, no. 1, pp. 2268-2271, 2001.

[69] S. A. Gourlay, "High field magnet R\&D in the USA," IEEE Trans. Appl. Supercond., vol. 14, no. 2, pp. 333-338, 2004.

[70] R. Gupta et al., "Status of high temperature superconducting magnet R\&D at BNL," IEEE Trans. Appl. Supercond., vol. 14, no. 2, pp. 1198-1201, 2004.

[71] J. Cozzolino et al., "Magnet engineering and test results of the high field magnet R\&D program at BNL," IEEE Trans. Appl. Supercond., vol. 13, no. 2, pp. 1347-1350, 2003.

[72] A. V. Zlobin et al., "Development of $\mathrm{Nb}_{3} \mathrm{Sn}$ accelerator magnets at Fermilab," to appear in these proceedings.

[73] _ - "Development and test of $\mathrm{Nb}_{3} \mathrm{Sn}$ cos-theta dipoles based on PIT strands," to appear in these proceedings.

[74] A. D. McInturff et al., "Test results for a high field (13 T) $\mathrm{Nb}_{3} \mathrm{Sn}$ dipole," in Proc. 1997 Part. Accel. Conf., 1998, pp. 3212-3214.

[75] R. Benjegerdes et al., "Fabrication and test results of a high field, $\mathrm{Nb}_{3} \mathrm{Sn}$ superconducting racetrack dipole magnet," in Proc. 2001 Part. Accel. Conf., 2001, pp. 208-210.

[76] A. F. Lietzke et al., "Test results of $\mathrm{HD} 1 \mathrm{~b}$, an upgraded 16 tesla $\mathrm{Nb}_{3} \mathrm{Sn}$ dipole magnet," to appear in these proceedings.

[77] P. McIntyre, "12 T hybrid block-coil dipole for future hadron colliders," IEEE Trans. Appl. Supercond., vol. 11, no. 1, pp. 2264-2267, 2001. 